\title{
Thermal Evolution of Magnetic Interactions in Ni Nanowires Embedded in Polycarbonate Membranes by Ferromagnetic Resonance
}

\author{
A. Ghaddar ${ }^{a}$, J. Gieraltowski ${ }^{a}$, F. Glohguen ${ }^{b}$, R. Żuberek ${ }^{c}$, P. Aleshievych ${ }^{c}$, \\ J. KazmierczaK ${ }^{c}$, A. Slawska-Waniewska ${ }^{c}$ And H. SzymczaK ${ }^{c, *}$ \\ ${ }^{a}$ Laboratoire de Magnétisme de Bretagne (UBO) — CNRS FRE 2697, 93837 Brest Cedex 3, France \\ ${ }^{b}$ Laboratoire de Chimie, Electrochimie Moléculaire et Chimie Analytique (UBO) - CNRS \\ UMR 6521, 93837 Brest Cedex 3, France \\ ${ }^{c}$ Institute of Physics, Polish Academy of Sciences, al. Lotników 32/46, 02-668 Warsaw, Poland
}

(Received October 26, 2009)

\begin{abstract}
$\mathrm{Ni}$ polycrystalline nanowires with diameters of 50, 80, and $100 \mathrm{~nm}$ were electrodeposited in cylindrical pores of track-etched polycarbonate membranes. Their magnetic properties were determined as a function of temperature using ferromagnetic resonance and magnetization measurements. At room temperature, the uniaxial anisotropy is equal to the shape anisotropy whereas an additional contribution is evidenced at low temperature. This additional contribution is attributed to magnetoelastic effects induced in the nanowires due to the different thermal expansion constants of $\mathrm{Ni}$ and polycarbonate. The analysis of magnetization processes in Ni nanowire arrays evidenced strong dipolar interactions inside the wires due to the domain structure. The coercive field of the nanowires was shown to be nearly a linear function of the temperature and could be accounted for temperature dependence of the uniaxial anisotropy.
\end{abstract}

PACS numbers: $62.23 . \mathrm{Hj}, 75.80 .+\mathrm{q}, 75.30 . \mathrm{Gw}$

\section{Introduction}

Ferromagnetic nanowires have recently been intensively studied due to their potential application in high density perpendicular recording media and magnetoelectronic systems [1]. At the same time, the highly oriented arrays of magnetic nanowires are excellent model materials to study magnetism of low-dimensional systems. In order to understand magnetization processes in these polycrystalline soft magnetic materials one should consider interplay between the intergrain exchange coupling and magnetic dipolar interactions. Additionally, the shape anisotropy as well as the magnetoelastic anisotropy due to the internal stresses have also considerable effect on magnetic properties of the nanowire arrays. All these interactions determine the energy balance and the stability of magnetic system.

In this work two arrays of Ni nanowires of similar geometry embedded in different matrixes - a polycarbonate or aluminate nanoporous membranes - are studied. These matrixes are characterized by entirely different thermal expansion coefficients which allows us to eluci-

* corresponding author; e-mail: szymh@ifpan.edu.pl date the role of the magnetoelastic anisotropy in the overall magnetic behavior of Ni nanowire systems. The important role of the magnetoelastic effects for the nanowire arrays has been pointed already by Navas et al. [2]

\section{Experimental details}

The electrodeposition bath was prepared from analytical grade $\mathrm{Ni}\left(\mathrm{SO}_{4}\right) \cdot 7 \mathrm{H}_{2} \mathrm{O}(44 \mathrm{~g} / \mathrm{l})$ and $\mathrm{H}_{3} \mathrm{BO}_{3}(40 \mathrm{~g} / \mathrm{l})$, and deionized water. The nanowire samples were obtained by electrochemical reduction of the Ni salt into the pores of nuclear-track-etched polycarbonate membranes (Whatman) with one side covered by a sputter-deposited $\mathrm{Cu}$ layer (300 $\mathrm{nm}$ in thickness). The pore length was about $6 \mu \mathrm{m}$, and the pore diameters 50,80 , and $100 \mathrm{~nm}$, respectively (Fig. 1). Nickel electrodeposition was conducted at constant potential in a three-electrode configuration using an $\mathrm{Ag} / \mathrm{AgCl}$ reference electrode and a glassy carbon counter electrode. All the electrodeposition experiments were carried out at room temperature with no stirring. Monitoring the shape of the current vs. time response allowed us to stop the electrodeposition process before the covering of the membrane surface by a nickel layer [3]. Arrays of individual nickel nanowires were thus obtained, in which the majority of the wires tilts ran- 
domly of a few degrees with respect to the normal plane of the polycarbonate membrane. In a similar way the $\mathrm{Ni}$ nanowires in alumina films were obtained.

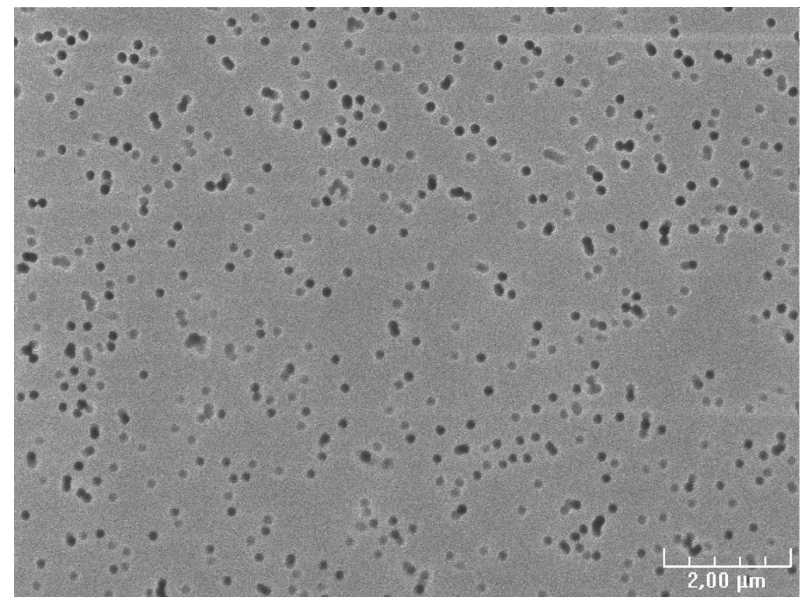

Fig. 1. Scanning electron microscopy (SEM) images of a nuclear-track-etched polycarbonate membrane with $80 \mathrm{~nm}$ pores, before $\mathrm{Ni}$ electrodeposition.

The ferromagnetic resonance (FMR) and magnetization investigations of $\mathrm{Ni}$ nanowires embedded in a polycarbonate membrane with the wire diameter of 50,80 and $100 \mathrm{~nm}$ and in the alumina films with diameter 20 and $100 \mathrm{~nm}$ have been performed. For this a standard electron paramagnetic resonance spectrometer (Bruker EMX-10/12) and standard vibrating sample magnetometer were used over the temperature range from liquid helium to room temperature (RT).

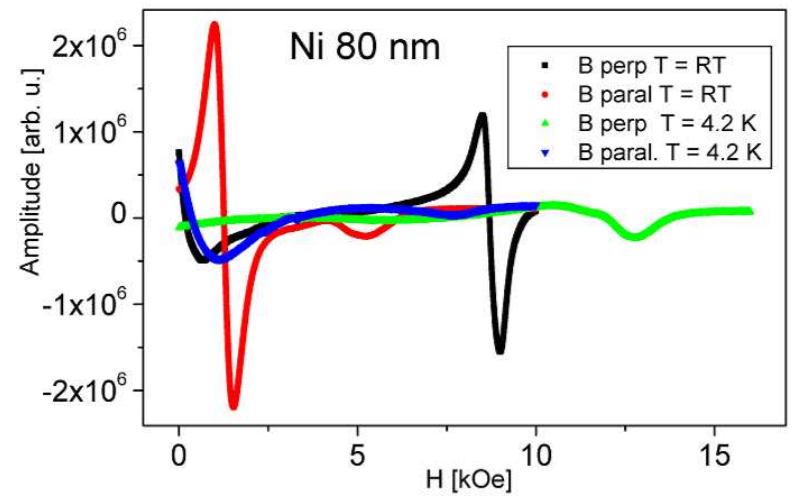

Fig. 2. Ferromagnetic resonance derivative spectra of $80 \mathrm{~nm}$ Ni nanowires embedded in polycarbonate matrix for parallel and perpendicular configuration at RT and $4.2 \mathrm{~K}$.

\section{Results and discussion}

The electron paramagnetic resonance spectrometer was employed in the FMR measurements. The sample was placed in the cavity that the angle $\left(\theta_{H}\right)$ between applied field $\left(H_{0}\right)$ and the wire axis can be adjusted from $0^{\circ}$ to $90^{\circ}$ with a perpendicular microwave pumping field $\left(h_{\mathrm{rf}}\right)$. All the measurements were performed at the frequency of $9.39 \mathrm{GHz}$ (X band). As examples the FMR derivative spectra for $80 \mathrm{~nm} \mathrm{Ni}$ nanowires for parallel and perpendicular orientations measured at $4.2 \mathrm{~K}$ and RT are shown in Fig. 2. Similar results were obtained for samples with 50 and $100 \mathrm{~nm}$ wires. Due to the absence of the bulk magnetocrystalline anisotropy, resulting from the polycrystalline structure of the nanowires, the geometry of the system is axial. Taking this into account and assuming uniform distribution of magnetization the resonant frequency, $\omega$, of the system can be obtained as [4-6]:

$$
\begin{aligned}
& \left(\frac{\omega}{\gamma}\right)^{2}=\left[H_{\text {eff }} \cos 2 \theta_{0}+H_{0} \cos \left(\theta_{0}-\theta_{H}\right)\right] \\
& \times\left[H_{\text {eff }} \cos ^{2} \theta_{0}+H_{0} \cos \left(\theta_{0}-\theta_{H}\right)\right],
\end{aligned}
$$

where $\gamma$ is the gyromagnetic ratio, $H_{\text {eff }}$ is an effective uniaxial field along the wires axis, which contains three main parts: the uniaxial magnetic anisotropy $\left(H_{\mathrm{A}}\right)$, the shape anisotropy of the wire itself $\left(2 \pi M_{\mathrm{S}}\right)$ and the dipolar coupling between wires $\left(6 \pi M_{\mathrm{S}} P\right) . \theta_{0}$ and $\theta_{H}$ are the angle between the wire axis and the magnetization $M$ and applied field $H_{0}$, respectively. The equilibrium angle $\theta_{0}$ is determined from the minimum of the free energy of the system. The dipolar interaction is expressed as an uniaxial mean field which can be considered proportional to the porosity $P=(2 \pi / \sqrt{3})(R / d)^{2}[6]$ where $R-$ radius of the wire and $d$ is the inter-wire distance. The effective field taken from the shape demagnetization is $2 \pi M_{\mathrm{S}}$. Then, for effective field we have

$$
H_{\text {eff }}=H_{\mathrm{A}}+2 \pi M_{\mathrm{S}}-6 \pi M_{\mathrm{S}} P \text {. }
$$

In our case the porosity is very small $(1 \%$ for $50 \mathrm{~nm}$ wires and $2 \%$ for $80 \mathrm{~nm}$ wires). Therefore the dipolar interaction between nanowires is very small in comparison with other terms and its contribution to the effective field will be neglected. In this analysis no domain structure was taken into account. It is oversimplification, because additional low field microwave absorption seen in Fig. 2 gives experimental evidence for a presence of domains in $\mathrm{Ni}$ wires [6]. Such behaviour is expected, since the polycrystalline nanowires cannot be the single domain objects. This conclusion is confirmed in this paper using the Henkel plot (see Eq. (5)).

The temperature dependence of the effective field $\left(H_{\text {eff }}\right)$ and the demagnetizing field $\left(2 \pi M_{\mathrm{S}}\right)$ for $50 \mathrm{~nm}$ wires are shown in Fig. 3. It is seen that the effective field strongly depends on the temperature in contrast to the demagnetizing field which is only weekly temperature dependent. Similar situation is observed for $80 \mathrm{~nm}$ (Fig. 4) and $100 \mathrm{~nm}$ wires. According to Eq. (1) it means that anisotropy field $H_{\mathrm{A}}$ should strongly depend on temperature. Figure 5 confirms such behaviour of $H_{\mathrm{A}}$ for all three samples. Generally, $H_{\mathrm{A}}$ in polycrystalline wires is expected to arise due to the surface anisotropy or due to magnetoelastic interactions. While the first mechanism is not effective, the second one seems to be able to explain $H_{\mathrm{A}}(T)$ dependence. It was presented in Refs. [7, 8] that the uniaxial anisotropy may be related to the presence of 


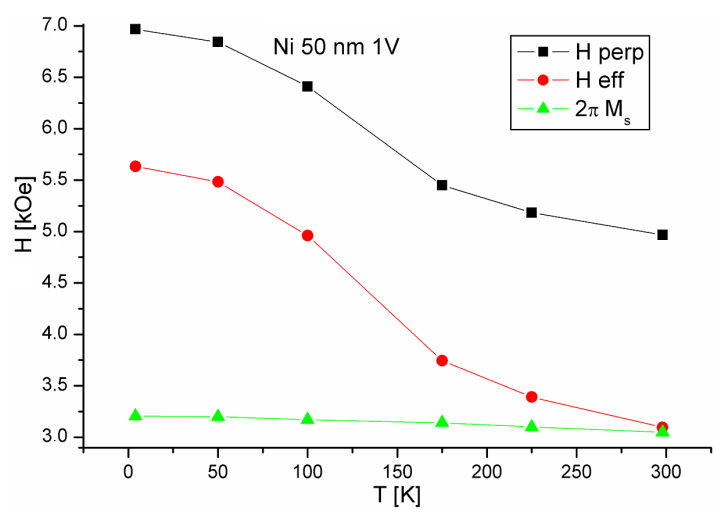

Fig. 3. Perpendicular resonance field, effective field and demagnetizing field $2 \pi M_{\mathrm{S}}$ for $50 \mathrm{~nm}$ Ni nanowires embedded in polycarbonate matrix.

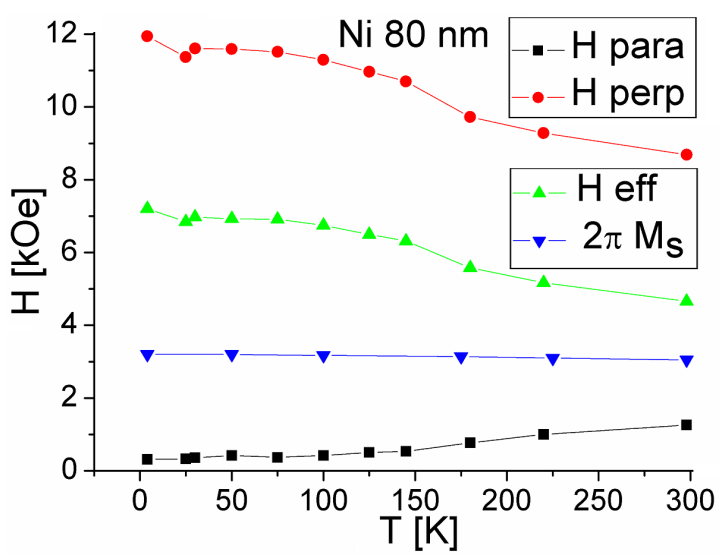

Fig. 4. Perpendicular and parallel resonance fields, effective field and demagnetizing field $2 \pi M_{\mathrm{S}}$ for $80 \mathrm{~nm} \mathrm{Ni}$ nanowires embedded in polycarbonate matrix.

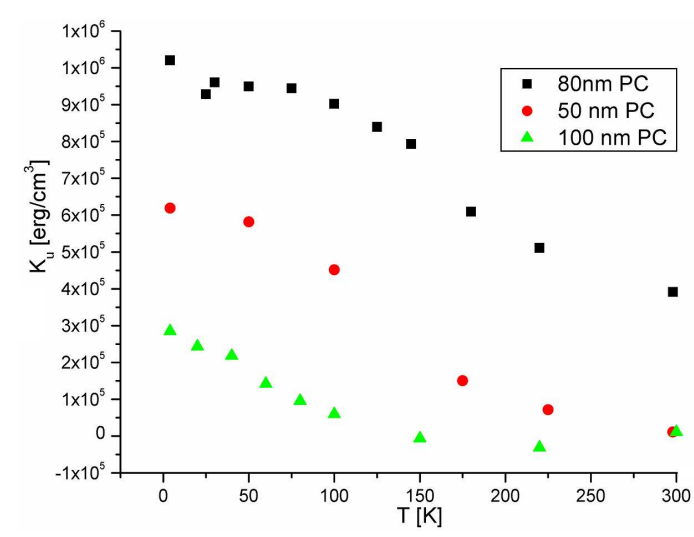

Fig. 5. Temperature dependence of the uniaxial anisotropy constants for Ni nanowires with diameters of 50,80 , and $100 \mathrm{~nm}$ embedded in polycarbonate matrix. the polycarbonate membrane and to the chemical bonding existing between polycarbonate matrix and $\mathrm{Ni}$ wires. The thermal stresses induced in the nanowires due to the different thermal expansion constants of the Ni and polycarbonate result in magnetostrictive effects which should be taken into account. These Ni deformations, induced upon temperature changes, have their consequences in the magnetoelastic energy. For the polycrystalline Ni wires this energy can be written as [9-11]:

$$
E_{\mathrm{me}}=-(3 / 2) \lambda_{\mathrm{s}}\left(\sigma_{r r} \alpha_{r r}^{2}+\sigma_{\varphi \varphi} \alpha_{\varphi \varphi}^{2}+\sigma_{z z} \alpha_{z z}^{2}\right),
$$

where $\lambda_{\mathrm{s}}$ is saturation magnetostriction constant, $\sigma_{i i}$ are the diagonal components of the residual stress tensor in cylindrical coordinates and $\alpha_{i i}$ are the components of the unit magnetization vector. The stress depends on the ratio of linear expansion coefficients of polycarbonate and $\mathrm{Ni}$. It could be calculated according to the Dubois et al. model [8] and it is responsible for the temperature dependence of the $H_{\mathrm{A}}$.

It is confirmed additionally by the temperature dependence of magnetic anisotropy in $\mathrm{Ni}$ wires embedded in aluminate. The linear expansion of the aluminium oxide (sapphire) is smaller than that of nickel (for the nickel it is $13.0 \times 10^{-6} / \mathrm{K}$ and for alumina it is $8.4 \times 10^{-6} / \mathrm{K}$ ) and therefore the temperature dependence of magnetic anisotropy has different sign compared to the wires embedded in polycarbonate matrix (the average thermal expansion coefficient for polycarbonate is equal to $\left.65.0 \times 10^{-6} / \mathrm{K}\right)$. It is shown in Fig. 6. These results are similar to these obtained by Navas et al. [2].

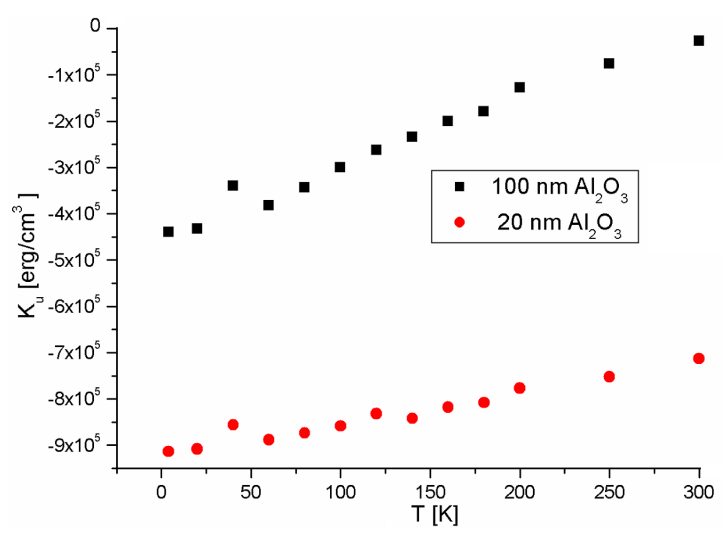

Fig. 6. Temperature dependence of the uniaxial anisotropy constants for $\mathrm{Ni}$ nanowires with diameters of 20 and $100 \mathrm{~nm}$ embedded in aluminate.

The stress dependence of the magnetic anisotropy was confirmed by the magnetization measurements. Hysteresis loops have been measured using the vibrating sample magnetometer with an external magnetic field applied parallel or perpendicular to the wire axis in the temperature range from the liquid helium up to the room temperature. Figure 7 presents the hysteresis loops for two temperatures: $4.2 \mathrm{~K}$ and the room temperature. The temperature dependence of the coercive field, $H_{\mathrm{C}}$, is shown in Fig. 8. The $H_{\mathrm{C}}(T)$ is practically a linear function of 


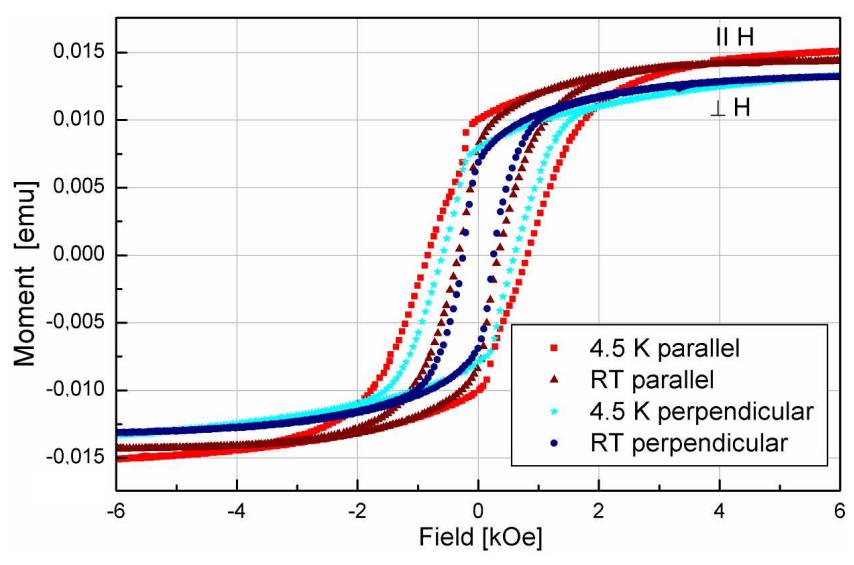

Fig. 7. Hysteresis loops for an assembly of $\mathrm{Ni}$ nanowires with diameter of $80 \mathrm{~nm}$ embedded in polycarbonate matrix at $4.2 \mathrm{~K}$ and RT. The applied field was oriented parallel and perpendicular to the wires.

temperature. Similar temperature dependence of the coercive field was observed in [7]. This is in contrast with predictions of models of nucleation by thermal activation over an anisotropy barrier, leading to a $T^{1 / 2}$ variation. Even if a reasonable distribution of barriers is assumed, the temperature dependence is still reminiscent of a $T^{1 / 2}$ dependence [12]. The observed here temperature dependence of the coercive field suggests that it is dominated by magnetostrictive effects. The coercive field can be estimated by [13]:

$$
H_{\mathrm{C}}=3\left|\lambda_{\mathrm{s}} \sigma\right| / M_{\mathrm{S}} \text {. }
$$

According to this model the coercivity $H_{\mathrm{C}}(T)$ should be proportional to $K_{U}(T)$. The results presented in Figs. 7 and 8 confirm the expected dependence and indicate that the magnetoelastic interactions play important role in determining the magnetic properties of $\mathrm{Ni}$ nanowires embedded in polycarbonate membranes.

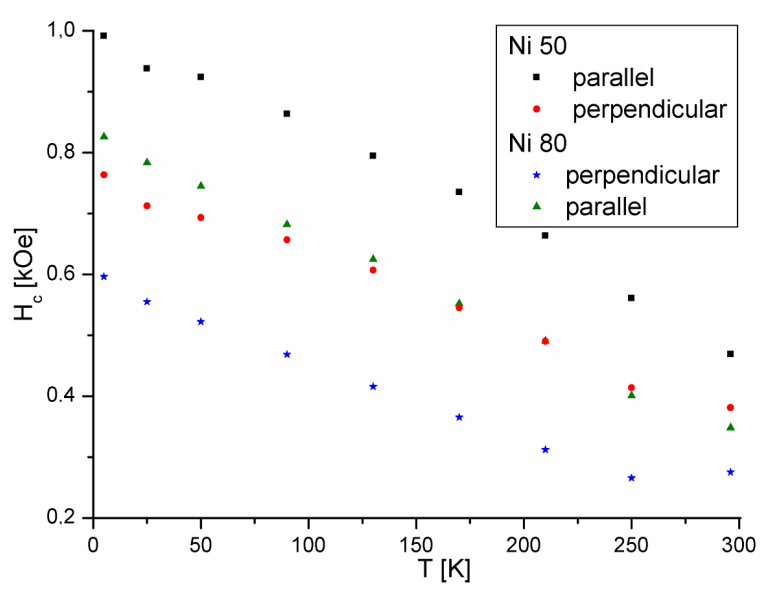

Fig. 8. Temperature dependence of the coercive field for $\mathrm{Ni}$ nanowires with diameters of 50 and $80 \mathrm{~nm}$ embedded in polycarbonate matrix.

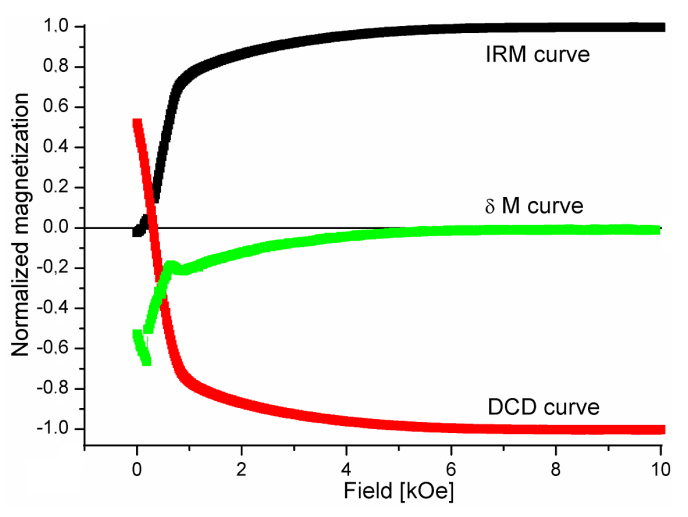

Fig. 9. Isothermal remanent magnetization $M_{\mathrm{IRM}}(H)$ curve, DC demagnetization remanence $M_{\mathrm{DCD}}$ curve, and $\delta M(H)$ curve for $50 \mathrm{~nm} \mathrm{Ni}$ nanowires embedded in polycarbonate matrix.

Another types of interactions responsible for magnetic properties of $\mathrm{Ni}$ nanowires are interactions between the grains. Generally, there are two types of such interactions: the intergrain exchange interactions and the dipolar interactions. In order to obtain information about relative role of these interactions the isothermal remanent magnetization $M_{\mathrm{IRM}}(H)$ and the DC demagnetization remanence $M_{\mathrm{DCD}}(H)$ have been measured (Fig. 9). According to $[14,15]$ the $\delta M(H)$ curve (the Henkel plot)

$$
\delta M(H)=M_{\mathrm{DCD}}(H)-\left[1-2 M_{\mathrm{IRM}}(H)\right]
$$

may be used to evidence what type of interaction is dominant in a magnetic system. Positive peak indicates intergrain exchange interactions, while negative peak indicates dipolar interactions between individual wires or inside the wires. As shown in Fig. 9, the large negative peak shows that there is a strong dipolar interaction and no exchange coupling interaction in this medium. But it is unlikely that this effect is due to the dipolar interactions between wires, as the porosity in the studied system is very low. It is possible that this effect is caused by the domain structure within each wire.

\section{Conclusions}

The FMR and magnetization measurements of $\mathrm{Ni}$ nanowires embedded in polycarbonate matrix have been analyzed in order to obtain information on the effects of the competing interactions on magnetic properties of the system. At room temperature, the uniaxial anisotropy is dominated by the shape anisotropy whereas an additional anisotropy is evidenced at low temperatures. This additional uniaxial anisotropy has the magnetoelastic origin because of the mismatch of the matrix - and Ni thermal expansion coefficients. The analysis of the magnetization processes in Ni nanowire arrays evidences strong dipolar interactions inside the wires and no intergrain exchange interactions. The strong dipolar interactions are due to the domain structure within each wire. 


\section{Acknowledgments}

This work was partially supported by the Ministry of Education and Science (Poland) under the Polish Network of "New materials and sensors for optoelectronics, information technology, energetics and medicine" as well as Research Project 4530/B/T02/2008/34.

\section{References}

[1] D. Appell, Nature 419, 553 (2002).

[2] D. Navas, K.R. Pirota, P. Mendoza Zelis, D. Velazquez, C.A. Ross, M. Vazquez, J. Appl. Phys. 103, 07D523 (2008).

[3] L. Sun, Y. Hao, C.-L. Chien, P.C. Searson, IBM J. Res. Dev. 49, 79 (2005).

[4] A. Encinas-Oropesa, M. Demand, L. Piraux, I. Huynen, U. Ebels, Phys. Rev. B 63, 104415 (2001).

[5] U. Ebels, J.-L. Duvail, P.E. Wigen, L. Piraux, L.D. Buda, K. Ounadjela, Phys. Rev. B 64, 144421 (2001).

[6] T. Li, Y. Sui, Z. Huang, S. Yang, B. Gu, Y. Du, J. Phys., Condens. Matter 17, 3637 (2005).
[7] J. Meier, B. Doudin, J.-Ph. Anserment, J. Appl. Phys. 79, 6010 (1996).

[8] S. Dubois, J. Colin, J.L. Duvail, L. Piraux, Phys. Rev. B 61, 14315 (2000).

[9] N. Usov, A. Antonov, A. Dykhne, A. Lagar'kov, J. Phys., Condens. Matter 10, 2453 (1998).

[10] A.S. Antonov, V.T. Borisov, O.V. Borisov, A.F. Prokoshin, N.A. Usov, J. Phys. D 33, 1161 (2000).

[11] R. Zuberek, H. Szymczak, M. Gutowski, A. Zhukov, V. Zhukova, N.A. Usov, K. Garcia, M. Vazquez, J. Magn. Magn. Mater. 316, e890 (2007).

[12] R.W. Chantrell, K. O'Grady, A. Bradbury, S.W. Charles, J. Popplewell, J. Phys. D 18, 2505 (1985).

[13] C.-W. Chen, in: Magnetism and Metallurgy of Soft Magnetic Materials, North-Holland, Amsterdam 1977.

[14] J. Yuan, W. Pei, T. Hasagawa, T. Washiya, H. Saito, S. Ishio, H. Oshima, K.-I. Itoh, J. Magn. Magn. Mater. 320, 134 (2008).

[15] O. Henkel, Phys. Status Solidi B 7, 919 (1964). 